\title{
Akupunktur scheint bei Fibromyalgie zu helfen
}

\author{
Die Akupunktur an individuell gewählten Punkte lindert bei Fibromyalgiepatienten gegenüber \\ einer Scheinmaßnahme signifikant die Schmerzen und verbessert die Lebensqualität.
}

\begin{abstract}
_ Langfristig gibt es kaum einen Fibromyalgiepatienten, der nicht auch einmal zu alternativmedizinischen Methoden Zuflucht gesucht hätte. In einer randomisierten, kontrollierten, multizentrischen Studie wurde nun bei 164 Patienten mit Fibromyalgie der Effekt von zehn Akupunktursitzungen einmal pro Woche untersucht. Bei der Verumgruppe wurden Punkte nach den Regeln der traditionellen chinesischen Medizin (TCM) gestochen, in der Placebogruppe wurde nur die Nadelhülle ohne Nadel an definierten Punkten am Rücken und in der Lumbalregion eingesetzt. Die Effekte wurden zu Beginn der Studie, nach zehn Wochen sowie nach sechs und zwölf Mo-
\end{abstract}

naten verblindet evaluiert. Für die Schmerzen wurde eine visuelle Analogskala eingesetzt, für Depression und Lebensqualität die Hamilton-Skala und der Fragebogen SF-12.

Eine Intention-to-treat-Analyse ergab nach zehn Wochen einen hoch signifikanten Rückgang der Schmerzen von 41\%. Langfristig nutzte sich der Effekt etwas ab, lag jedoch auch nach zwölf Monaten noch bei knapp 20\%. Somit lag die Effektstärke wesentlich höher als z. B. bei Pregabalin oder Duloxetin. Auch bei der Lebensqualität war eine signifikante Verbesserung festzustellen, vor allem im physischen Bereich. Im Hamilton-Score wurde nur eine Reduktion um 25\% be- obachtet, die sich nicht signifikant von der Scheingruppe unterschied.

- Vas J et al. Acupuncture for fibromyalgia in primary care: a randomised controlled trial. Acupunct Med. 2016;34:257-66

\section{KOMMENTAR}

Bezüglich der Studienqualität setzt die Arbeit neue Maßstäbe. Der im Vergleich zu früheren Studien enorm günstige Effekt geht wohl auch auf die Behandlung durch erfahrene Therapeuten zurück. Auch wenn das theoretische Gebäude der TCM für westlich geschulte Ärzte nur schwer nachvollziehbar ist, so ist man gerade bei der Fibromyalgie für jede Alternative dankbar, da wir bisher nur wenig Effektives anzubieten haben.

Prof. Dr. med. H. S. FüeßI

\section{Acanthosis nigricans und Hyperandrogenismus}

Eine 27-jährige Frau stellte sich wegen seit zwei Jahren bekannter Acanthosis nigricans (Abb. A, B), Hirsutismus und Amenorrhö vor. Der Insulinspiegel lag bei über $200 \mu \mathrm{U} / \mathrm{ml}$ (Norm: $2-13 \mu \mathrm{U} / \mathrm{ml}$ ), das Plasma-Testosteron war mit $649 \mathrm{ng} / \mathrm{ml}$ massiv erhöht, es fanden sich Antikörper gegen Thyreoperoxidase (TPO) sowie antinukleäre Antikörper (ANA) mit einem Titer von 1:64. Die Werte für Blutzucker und $\mathrm{HbA}_{1 \mathrm{c}}$ waren normal, eine Malignomsuche blieb negativ.

Bei der Wiedervorstellung nach sechs Monaten hatte die Patientin deutlich an Gewicht verloren. Nüchtern ergab sich ein Blutzuckerwert von 174 $\mathrm{mg} / \mathrm{dl}$, der $\mathrm{HbA}_{1 \mathrm{c}}$-Wert lag bei 11,2\% . Der C-Peptid-Spiegel war mit 7,3 ng/ $\mathrm{ml}$ leicht erhöht, der Test auf Insulin-Antikörper negativ. Man begann eine Therapie mit Metformin, unter der sich sechs Monate später Hypoglykämien einstellten. Der Titer der Insulinrezeptor-Antikörper war mit 1:1.280 erhöht. Unter der Diagnose eines Insulinresistenzsyndroms vom Typ B begann man eine Therapie mit Prednisolon und Azathioprin, worunter sich binnen sechs Wochen Blutzucker, Testosteron und Insulinspiegel normalisierten und die Hautbefunde verbesserten (Abb. C, D).

Eine Acanthosis nigricans kann durch endokrine, metabolische, genetische und paraneoplastische Faktoren hervorgerufen werden. Das Insulinresistenzsyndrom Typ B ist eine seltene Autoimmunerkrankung, bei der Antikörper gegen den Insulinrezeptor zirkulieren, die zu Störungen des Blutzuckerstoffwechsels, extremer Insulinresistenz, Acanthosis nigricans und Hyperandrogenismus führen. Hier ist der Spezialist gefragt.

Prof. Dr. H. S. FüeßI - Lauria MW, Saad MJ (marciowlauria@gmail.com). Acanthosis nigricans and insulin resistance. NEnglJ Med. 2016;374:e31
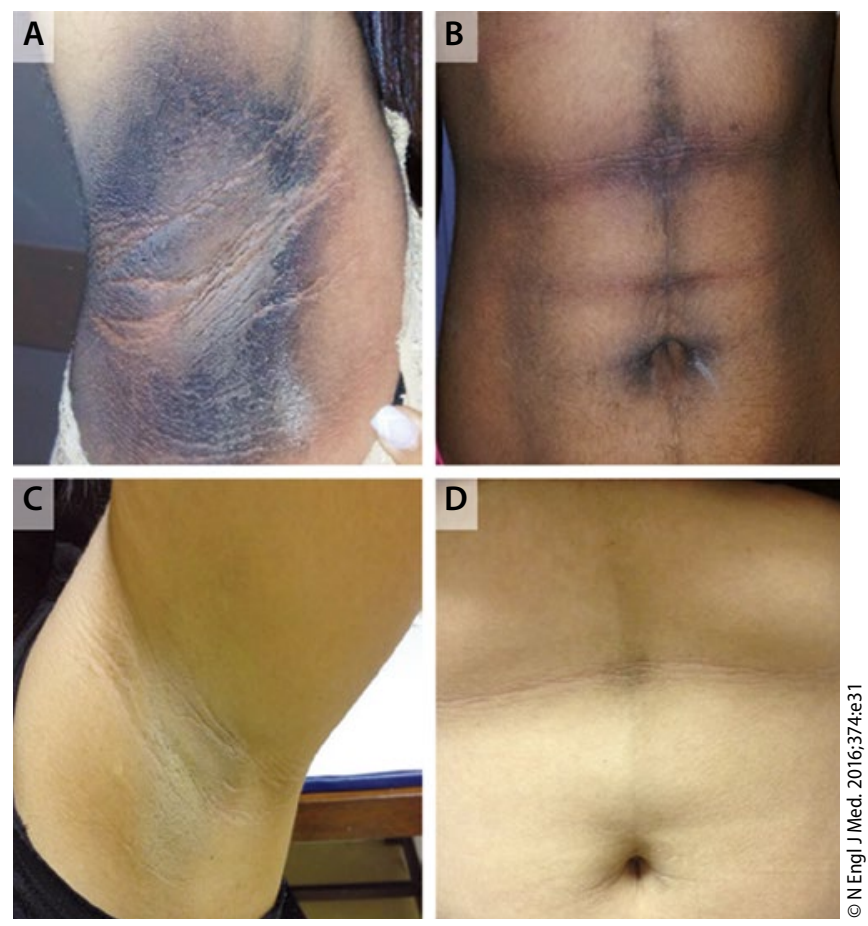

A, B: Acanthosis nigricans bei einer 27-jährigen Frau.

C, D: Zustand nach sechs Wochen Arzneimitteltherapie. 